Horizons philosophiques

\title{
Entretien avec Gaétan Soucy : confidences sur l'écritoire
}

\section{Luc Abraham}

Volume 10, numéro 1, automne 1999

Écritures et confessions

URI : https://id.erudit.org/iderudit/801103ar

DOI : https://doi.org/10.7202/801103ar

Aller au sommaire du numéro

\section{Éditeur(s)}

Collège Édouard-Montpetit

\section{ISSN}

1181-9227 (imprimé)

1920-2954 (numérique)

Découvrir la revue

Citer ce document

Abraham, L. (1999). Entretien avec Gaétan Soucy : confidences sur l'écritoire. Horizons philosophiques, 10(1), 1-13. https://doi.org/10.7202/801103ar d'utilisation que vous pouvez consulter en ligne.

https://apropos.erudit.org/fr/usagers/politique-dutilisation/ 


\section{ENTRETIEN AVEC GAÉTAN SOUCY : CONFIDENCES SUR L'ÉCRITOIRE}

Luc Abraham - Selon vous, écrire procède-t-il d'un geste égoïste ou, au contraire, généreux? Je m'explique : quand vous posez l'acte d'écrire, estce par désir d'immortalité, de puissance, ou dans un abandon gratuit?

GaÉTAN Soucr - C'est difficile de répondre à cela parce qu'il faudrait avoir davantage de lumière sur soi pour ne pas pouvoir se tromper. Mais j'ai l'impression que c'est un petit peu le miracle de l'art, dans la mesure où cela procède à la fois d'un égotisme foncier, peut-être même d'une volonté de puissance. Mais le résultat est tout de même un don : on fait présent de quelque chose - que ce présent soit apprécié ou pas, c'est une autre affaire. J'ai l'impression que cela se situe dans un autre ordre en fait, parce que cela nous apparaît présenter une alternative autre que générosité versus égoïsme. Je pense qu'il y a un appel : on répond à une nécessité qui, sans vouloir mettre de la mystique inutile dans la chose, semble venir d'ailleurs que soi et concerner autre chose que soi. II y a aussi qu'il faut toujours maintenir en vie l'esprit, l'activité langagière des êtres humains; il faut bien qu'il y en ait qui s'en chargent. Je crois beaucoup à cette idée qu'écrire correspond à un appel. On entend quelque chose qui tire la parole de nous, qui nous appelle à dire. C'est peut-être une illusion, c'est sans doute une illusion; on pourrait toujours dépister les motifs psychologiques plus ou moins inconscients qui nous amènent à cette névrose-là et qui consiste à investir l'ensemble de la signification de sa vie dans l'acte d'écrire. II y a donc cet aspect psychologique aussi. Mais je persiste à croire qu'il y a quelque chose de plus dans l'écriture que la seule volonté de rayonnement du sujet psychologique.

- Donc, ce désir d'immortalité vous ne le ressentez pas?

- Si, si. Mais cela relève davantage de la névrose; Sartre a écrit un texte où la plupart des écrivains se reconnaissent, je pense, où il situe bien l'origine existentielle de cette névrose. C'est-à-dire qu'écrire consiste à croire qu'on va pouvoir se sauver par les mots. C'est une illusion, comme 
je disais. Mais sans cette illusion, il n'y aurait pas de littérature non plus. D'ailleurs, qui sait si on n'est pas sauvé d'une certaine manière, après tout? II y a une part de mystère là-dedans.

- Cela me fait penser à ce qu'Hemingway disait "écrire de son mieux, c'est se condamner à la solitude"; ainsi, l'écriture serait un repli sur soi préfigurant une ouverture à l'autre, vers les autres : vers un lectorat. C'est ce que vous me dîtes un peu...

- Oui. C'est ce paradoxe curieux d'un retour vers soi qui m'amène à l'autre. Je suis en train de lire en ce moment quelque chose qui est à la fois lancinant à lire et passionnant. C'est d'un auteur relativement peu connu, bien qu'il ait ses fanatiques : Claude Louis-Combet. II a écrit un livre qui s'intitule Le recours au mythe. II s'agit d'une sorte de biographie intérieure où il tente de cerner les motifs profonds qui l'ont amené à écrire, et il a écrit une œuvre considérable : une vingtaine d'ouvrages. C'est une littérature très cruelle. Ce texte est d'un narcissisme infini : il y a un repliement constant sur soi. Cependant, quelque chose jaillit de cela. C'est-à-dire que, curieusement, on se sent concerné, on se sent inspiré par ce narcissisme. II a une telle façon de parler de lui-même, de son expérience, qu'on s'y sent engagé soi-même totalement. Ça me semble être justement encore une fois le propre de l'écriture. Alors, je ne veux pas céder aux facilités concernant le singulier et l'universel - «plus je suis singulier et plus je suis universel» - , mais il est certain qu'il y a de ça. C'est de l'ordre de l'engagement encore. Jean-Paul Sartre disait que pour devenir un grand écrivain, ce n'est pas compliqué, il suffit de ne faire que cela : écrire. Montherlant disait "tout ce qui ne me passionne pas m'ennuie». Cet investissement dans l'écriture doit être total. Ce qui fait d'ailleurs souvent des écrivains des personnes insupportables. Constant avait cette phrase extrêmement lucide : "Je ménage les autres, mais je ne les aime pas". Je ne prendrais pas pour moi cette phrase là, je pense que j'aime les gens. Mais, c'est pour dire qu'il y a quelque chose d'un engagement exclusif dans l'écriture et c'est absolument le prix à payer. Et c'est d'autant plus douloureux qu'on n'est jamais certain d'y trouver son compte; il se peut qu'on ne produise que des choses de peu de signifiance, mais sans cet engagement, il n'y aurait pas de grands livres. C'est donc une condition nécessaire, sinon suffisante, hélas.

- L'écriture apporte-t-elle à l'écrivain que vous êtes un plaisir intense et premier par rapport auquel la publication devient secondaire. Être lu est-il moins important que l'acte d'écrire? 
- Il s'agit de deux ordres. S'il n'y avait pas ce sentiment d'intensité lors de l'écriture, je ne pense pas qu'on se décarcasserait à ce point. II y a des moments que j'ai connus en écrivant que je n'échangerais contre rien au monde. Par contre, il y a tout de même un moment nécessaire qui est celui du lecteur. Ça c'est très curieux, parce que j'ai déjà constaté une chose : une mauvaise critique vous atteint dans votre personne, alors qu'une critique élogieuse, c'est comme si on vantait les mérites de votre passeport. On ne se sent pas directement concerné; on a l'impression qu'on s'adresse à quelqu'un d'autre lorsqu'on s'adresse élogieusement à notre travail. Alors qu'une critique négative habituellement nous blesse dans notre personne. C'est curieux...

- C'est peut-être l'aspect narcissique de l'écrivain qui est touché lorsque cette critique n'est pas dithyrambique? Mais, cette conscience de l'écrivain envers son lectorat, vous impose-t-elle d'apporter une dimension politique, morale ou philosophique à votre travail? En fait, derrière la fiction, $y$ auraitil consciemment - puisque votre œuvre est à la fois fiction et réalisme - un message que vous souhaitez communiquer en le travestissant par le littéraire?

- Alors là, sûrement non. C'est d'ailleurs une tendance dont il faut profondément se méfier. On ne doit pas se servir de la fiction pour exprimer une idée, une certaine conception des choses, je ne sais trop... Je ne fais pas de politique en écrivant, sauf que je ne peux ignorer que ce que j'écris porte du sens, est porteur de sens. Mais il faut toujours que ce sens là me précède; William Faulkner, par exemple, a écrit une œuvre considérable dans les années trente. Et l'œuvre de Faulkner a périclité le jour où il a voulu dans son roman Parabole essayer justement d'exprimer une conception très noble de l'humanité, du courage et de la mystique humaniste. Le résultat est très peu probant par rapport à cette matière en fusion qui émanait de lui dans les années trente. Alors, je me méfie beaucoup de cela; j'essaie le plus possible de ne pas tenir compte du lecteur quand j'écris. Non par indifférence, mais par exigence créatrice interne. Parce que, si tant est que j'ai quelque chose à dire à mon lecteur, il faut que je ne tienne pas compte de lui. C'est curieux parce que le message n'acquiert une véritable efficacité que si l'on ne tient pas compte du locutaire. C'est propre aussi à l'activité créatrice, je crois.

- Dans la question précédente, nous parlions de la critique et dans 
celle-ci du lectorat. En mai 1999, dans le journal La Presse, le chroniqueur Pierre Foglia avait adressé une critique négative quant à votre dernier ouvrage, La petite fille qui aimait trop les allumettes. II semblait dire que vous écriviez pour un public "élitiste" puisqu'il y a des références à Spinoza, etc.

- Oui, mais il se trahit lui-même. Je trouve que c'est un papier tout à fait odieux, et facile : c'est proprement démagogique comme attitude de sa part. Il y a des gens qui ont émis des réserves par rapport à mon travail et je respecte tout à fait ces réserves-là , car ce sont des gens qui expliquaient pourquoi. Alors que Foglia se trahit lui-même en évoquant les noms de Spinoza, du duc de Saint-Simon, etc., pour dire "c'est du roman pour snobinard", alors que Spinoza est cité à trois reprises dans le livre et la narratrice dit toujours : «Mais, je n'y comprends rien, je n'y ai pas saisi un seul mot". Mais Foglia s'attaquait au phénomène; en quelque sorte, c'était une forme de consécration : que Foglia prenne son papier pour parler de moi, c'était un peu le parallèle avec l'éloge de mon livre par Pierre Lepape dans Le Monde. Sauf que justement, c'est un texte qui sent beaucoup sa mauvaise foi parce qu'il ne s'attaque pas directement à l'œuvre elle-même, mais il attaque le lectorat, et c'est ce qui m'a le plus blessé. Ce n'est pas tellement parce qu'il attaque mon livre, puisqu'il avoue d'emblée qu'il ne l'a pas lu, qu'il l'a lâché au bout de cinquante pages, mais c'est qu'il affirme : "Ceux qui prétendent aimer ça, ce sont des menteurs, ce sont des prétentieux, ce sont des hypocrites". Nathalie Pétrowski a été dans la même foulée et c'est tout aussi peu justifié. Mais je pense que cela relève d'un vieux malaise québécois, c'est-à-dire qu'on n'aime pas que quelqu'un émerge du troupeau. ${ }^{1}$

- On pourrait faire un lien avec toute la dimension culturelle du Québec, toute la culture populaire, à savoir effectivement que ce qui apparaît comme une pensée qui se développe est quelque chose de suspect.

- Oui, c'est une attitude démagogique anti-intellectualiste, carrément.

- Je n'insisterai pas là-dessus... Dans un autre ordre d'idée, votre style s'impose-t-il nécessairement en fonction du sujet traité : de L'Immaculée conception à La petite fille qui aimait trop les allumettes, $y$ a-t'il eu, selon vous, un changement de style ou de ton? J'ai l'impression que La petite fille a un style différent?

1. Au moment d'aller sous presse, nous apprenions que Pierre Foglia s'était rétracté. II dit maintenant, après relecture, avoir «beaucoup, beaucoup aimé». La Presse, samedi 28 août 1999. 
- Oui, comme vous le mentionniez à l'instant, le style est imposé pour moi par le livre même. Je n'ai pas trouvé une certaine façon d'écrire dans laquelle je me cantonnerai et dans laquelle je produirai toute mon œuvre. Je n'ai rien contre cela : on peut faire des oeuvres admirables de cette façon. On pense à Thomas Bernhardt, par exemple : c'est la même voix que l'on reconnaît de livre en livre. Ici, il y a Réiean Ducharme... Mais ce n'est pas ma façon de procéder : le style, pour moi, est commandé par l'histoire même que j'ai à raconter. Mais je le dis comme ça, comme si l'histoire préexistait à son style. Alors que c'est un phénomène étrange, il y a une dialectique temporelle là-dedans : l'un commande l'autre, l'histoire impose un style, mais le style aussi impose l'histoire. C'est assez difficile. La seule excuse, si tant est que j'ai besoin de me justifier de la chose, la seule excuse ce serait de dire que justement j'attends que surgisse en moi l'urgence d'une histoire et puis elle commande d'elle-même son style... Mais, cela dit, je crois qu'une lecture attentive et non prévenue de mon travail révélerait, je crois, une unité profonde non seulement sur le plan thématique - parce qu'il est beaucoup question de la faute, du pardon - mais aussi justement sur le plan du style. Sauf que c'est un style à vitesse variable, ou à géométrie variable. Quand dans quarante ans j'aurai clos ce que j'ai à dire, on verra peut-être surgir une unité inattendue et qui sera peut-être justement une unité stylistique.

- Vous évoquiez une urgence de l'histoire, mais parallèlement, y a-t-il chez vous une urgence d'écrire?

- Indéniablement. Le désir d'écrire n'est pas l'indice que l'on ait forcément quelque chose à dire, et c'est cela qui est terrible. Parce qu'il y a de vastes plages où je n'arrive pas à écrire et le désir est là, c'est d'ailleurs un désir qui peut prendre des formes paniques par moments. La petite fille a été écrite justement dans un moment comme celui-là, je me disais : "Je n'ai plus rien à dire". Je vivais cela comme une détresse, mais du fond de cette détresse a surgi ce texte qui a fait de moi un auteur célébré. C'est-à-dire que, jusqu'ici, j'étais un écrivain qui promettait, et là c'était comme si j'avais donné vraiment ce que j'avais à donner. Peu importe ce qu'on pense de la valeur littéraire de La Petite fille, il n'en demeure pas moins que c'est une expérience de vie assez unique que d'avoir écrit un texte comme ça. Et puis, cela nous vide aussi, beaucoup. Maintenant, j'hésite devant divers projets, je ne le dis pas pour faire dans l'anecdote, mais pour dire que justement ce désir d'écrire connaît ses failles. Je parlais d'appel tantôt : il faut 
écrire quand on se sent appelé par quelque chose, mais l'inverse ne fonctionne pas. On voudrait «appeler l'appel» : on appelle l'appel, mais quand il n'est pas là, rien à faire. Souvent il suffit de se mettre à son bureau, et les choses surgissent malgré soi. À ce titre, il faut se faire confiance.

- Vous écrivez le matin?

- Règle générale, j'écris le matin entre six heures et midi. Par contre, La petite fille - on l'a beaucoup dit - je l'ai écrit dans un temps très court. II a fallu que j'écrive à toute heure du jour. Je dormais à peu près deux heures par nuit, j'écrivais le matin, j'allais donner mes cours, je rentrais le soir, je faisais une sieste et puis je recommençais, parce que j'avais peur justement de perdre la voix. J'avais tellement l'impression d'être possédé par une voix, j'avais peur qu'elle me quitte. II fallait véritablement que je m'exécute, de peur de perdre cette voix. La peur de perdre la voix, chez l'écrivain, est analogue à la peur d'être enterré vivant.

- Alors, toujours en parlant de style... La culture japonaise, que vous connaissez bien je crois, la langue même de ce pays ont-elles eu une influence sur votre façon d'écrire, sur le regard que vous posez sur les choses et les êtres, sur la structure même de la langue que vous utilisez?

- Je pense que oui, mais je ne crois pas que cela a été de propos délibéré. Ce sont des constatations que j'ai pu faire a posteriori. Sans vouloir trop particulariser le débat, je crois que l'écriture en sait davantage que nous. L'écrivain véritable est celui qui est justement à l'écoute de ce qui n'a pas encore été révélé... J'ai de la difficulté avec les écrivains trop concertés, trop accomplis. Même s'il y en a certains parmi eux que j'admire beaucoup : Thomas Mann, Michel Tournier. Mais je crois qu'on ne doit pas savoir d'avance ce que doit contenir un texte, un texte doit toujours en savoir davantage que nous et, à ce titre, ce texte se nourrit d'événements biographiques - dans mon cas ce peut être mon expérience continue et profonde de la culture japonaise... Mais cela doit travailler de façon souterraine, de manière qui m'échappe jusqu'à un certain point, pour véritablement aboutir à quelque chose d'intéressant. Ainsi, je me suis rendu compte en discutant avec des amis japonais que mon second livre, L'Acquittement, avait quelque chose de profondément japonais. Et j'en ai d'ailleurs fait l'expérience avec une amie japonaise : ce livre, on peut le transposer à l'intérieur de la culture japonaise, et véritablement, tout colle, tout marche. De même dans mon dernier livre, dont on s'est beaucoup 
étonné de la syntaxe, je me suis rendu compte aussi, à posteriori, de l'influence qu'avaient eu la syntaxe ou la grammaire japonaise. Mais encore là, tout cela s'est fait inconsciemment, enfin de façon absolument non délibérée.

- Si votre style s'est nourri, même inconsciemment, d'une culture japonaise, ou de votre connaissance de la culture japonaise, en est-il de même pour votre inspiration? Je me rappelle que Maupassant se définissait comme un "regardeur". II disait que l'œil du romancier est "comme une pompe qui absorbe». Pensez-vous que ce regard vampirique soit le propre de l'écrivain puisant dans ce qui l'entoure les sources de son inspiration? Étant donné que vous avez vécu à plusieurs reprises au Japon, est-ce que le Japon n'a pas été source de votre inspiration?

- Certainement, mais encore une fois, je ne sais pas dans quelle mesure et je parviens mal à fixer le profil de l'influence que le Japon a pu exercer sur mon travail. Oui, je crois que Maupassant était un regardeur, et ce n'est d'ailleurs pas pour rien qu'il rapporte l'anecdote avec Flaubert qui lui dit : "Tu t'assois devant un arbre et tu le décris". II faut savoir regarder, mais je crois appartenir à une autre famille d'écrivains; je suis de ceux qui puisent en eux-mêmes, finalement, le référent de ce qu'il ont à dire. Cela ne veut pas dire que je n'observe pas, mais il n'y a pas un travail de transposition directe de ce que je peux observer. Véritablement, le texte en moi se prépare de lui-même, s'organise en moi et ensuite, il s'agit pour moi d'avoir l'oreille assez juste pour entendre ce que le texte a à me dire. C'est ma méthode de résolution de problèmes - parce que toute écriture de livre implique des problèmes : doit-on faire précéder telle scène par telle autre, comment se faire rencontrer tel personnage avec tel autre, comment amener tel élément ? Ces questions-là, je ne les résous jamais de manière conceptuelle mais toujours de manière intuitive, au sens où un musicien pourrait juger d'un timbre. Non pas en essayant de se référer à une quelconque théorie musicologique, mais en demandant à un violoniste et à un pianiste de jouer ces trois mesures-là ensemble, et puis juger à l'oreille (un ami mathématicien, parlant de ses recherches en théorie des nombres, qui tournaient en rond, me disait : "Je ne sais pourquoi, ça ne rime pas..."). II y a ce travail intuitif qui est pour moi extrêmement important; je crois que c'est ce que je peux dire de plus juste, de plus sincère sur mon travail. 
- Encore Maupassant, puisque vous aussi l'avez évoqué; il disait que pour écrire il faut "voir : (...) et voir juste. (c'est-à-dire) voir avec ses propres yeux et non avec ceux des maîtres". Vous qui avez une formation en philosophie - votre maitrise portait sur la philosophie transcendantale des sciences dans la philosophie critique kantienne -, pouvez-vous affirmer que votre écriture est "affranchie» par rapport à vos "maîtres"?

La philosophie a évidemment joué beaucoup dans la formation de ce que je suis, mais non pas directement dans la formation de mes textes en tant que telle. Mes textes, je me répète, fermentent en moi, se créent en moi, se créent à partir de ma matière. Or, il se trouve que dans ma matière première on trouve du Kant, du Spinoza, ma formation première en mathématiques et en physique. Mais tout cela en quelque sorte, joue d'un libre jeu. Et c'est d'ailleurs cela qui est pour moi intéressant au premier chef et qui fait la matière de la découverte; il y a bien sûr une part d'organisation structurale d'un livre - un livre ça se construit - mais ça se construit justement à partir d'une matière première. C'est que l'écrivain, contrairement au musicien ou contrairement au peintre, a à créer sa matière première. Un peintre part des couleurs - les couleurs préexistent - , pour le musicien, les sons préexistent. Alors que, pour le romancier, rien ne préexiste : il n'y a pas de matière première. On va dire : «les mots". Mais non! les mots sont là pour s'effacer. Le peintre nous présente des couleurs, pas pour que ces couleurs s'effacent : elles doivent être au premier plan. De même, pour le musicien, le son et son timbre doivent être au premier plan. C'est ça qui nous importe, c'est à cela qu'on a accès. Alors que pour un romancier, il ne faut justement pas que le lecteur s'arrête aux mots, il ne faut pas que le mot fasse écran par rapport à ce qu'il a à dire. Donc, le mot n'est pas une matière première comme la couleur ou le son peuvent l'être dans d'autres formes d'expression. II s'agit donc de la créer, cette matière première. Or, comment la crée-t-on bien? II s'agit d'ouvrir les valves; il y a un premier moment d'écriture sauvage où quelque chose surgit, s'organise de façon encore protozoaire. Puis, il y a un véritable travail artistique au sens artisanal du terme qui consiste à essayer d'organiser cette matière première. Mais cette matière première peut ne pas surgir, peut ne pas exister. II y a des gens qui sont de forts honnêtes artisans, pleins de bonne volonté, mais chez qui manque cette matière première : un manque d'imagination, un manque de sensibilité au monde, un manque d'expérience de vie, je ne sais trop. II y a quelque chose qui manque. L'exigence peut-être. Le «talent» n'est peut-être rien d'autre essentielle- 
ment qu'intransigeance vis-à-vis de soi.

- Avant d'entreprendre des études en littérature, vous avez poursuivi une formation en physique et en mathématiques, deux disciplines se posant comme des outils de savoir. Pensez-vous qu'on peut en dire autant de la littérature? La fiction peut-elle prétendre à être un outil de cognition ou n'en est-elle qu'un objet?

- La question est vaste, mais intéressante. Tout est affaire de vocabulaire, comme c'est souvent le cas. Si on définit la connaissance à partir des seuls critères épistémologiques de la scientificité, il est évident que Le procès de Kafka n'ouvre à aucune espèce de connaissance. Mais, voilà : est-ce que ce que l'être humain comprend et connaît se réduit aux seuls critères épistémologiques de la scientificité? C'est toute une question. Personnellement, je crois que la littérature - et le mot "outil» est peut-être un peu inadéquat en l'occurrence - est une voie de connaissance. Pour moi, c'est absolument certain. Je ne vois pas comment je pourrais nommer autrement que "connaissance" l'expérience que j'ai vécue en passant à travers l'œuvre de Kafka ou de Dostoïevski. J'ai connu quelque chose; quelque chose m'a fait grandir, m'a amélioré dans la compréhension que j'avais de moi-même et d'autrui, du rapport spirituel de l'humanité au monde. Il est certain qu'il y a là quelque chose qui est de l'ordre de la cognition, sauf que ce n'est évidemment pas une cognition qui se laisse réduire épistémologiquement à des critères scientifiques. Et je dirais la même chose de la philosophie : elle n'est évidemment pas là pour produire une axiomatique, découpant un objet à l'intérieur de la réalité et élaborant un système d'assertions logiquement reliées pour parler de cet objet. II est certain que la philosophie, ce n'est pas ce qu'elle a à faire. Mais sans la philosophie ne se poserait justement pas la question du rapport entre le sujet et l'objet qu'il essaie de définir. II faut toujours se souvenir que la connaissance peut et doit être autre chose qu'uniquement la connaissance scientifique. La connaissance scientifique est absolument nécessaire, indispensable, mais il ne faut pas la croire suffisante encore une fois, pour traduire l'expérience complexe du rapport de la conscience au monde.

- Vous avez évoqué Kafka. Avant d'être écrivain, vous avez été lecteur, vous l'êtes certainement encore. Quels ont été - quels sont encore - les livres qui vous ont marqué? Plus précisément, comme disait Kafka : "Un livre doit être la hache qui brise la mer gelée en nous". Quel a été ce livre pour vous Gaétan Soucy? 
- L'œuvre de Beckett a sans doute été chez moi l'expérience la plus importante sur le plan littéraire, mais pas juste sur le plan littéraire. Ce qui a fendu le bloc de glace en moi, comme le dit Kafka? II y a eu Victor Hugo quand j'avais treize-quatorze ans. Les travailleurs de la mer, ça vaut encore le détour...Puis, il y a eu Sartre. Alors, Sartre cela a été intéressant parce que j'ai connu son œuvre vers la fin du secondaire $V$, à quinze-seize ans. Je m'étais astreint à lire L'Etre et le néant parce que tout le monde disait autour de moi que c'était illisible : cela me provoquait à le lire. Tout comme lorsque je me suis mis à faire du japonais, c'est pour beaucoup parce que tous les textes que je lisais sur la culture japonaise indiquaient tout le temps à quel point c'était une langue infernale. Cela a piqué ma curiosité et ça a été la même chose pour L'Etre et le néant. Découvrir cela à seize ans, c'est quelque chose et j'ai vraiment fait un travail très très assidu: je négligeais mes cours de secondaire $V$ parce que je travaillais sérieusement trois heures par jour là-dessus, je fouillais dans les dictionnaires. Ça a été l'éveil philosophique pour moi, le premier grand texte de philosophie que j'ai lu. Et puis, il y a eu Beckett. Et parmi tout ça, il y a évidemment eu d'autres écrivains qui m'ont marqué nécessairement comme Dostoïevski, comme Kafka. Ou comme Albert Cohen, immense de beauté.

\section{- Poe?}

- Edgar Poe ça a été plutôt vers onze-douze ans; j'ai découvert Poe et ça m'avait beaucoup choqué au sens où j'avais éprouvé un choc profond. Mais si je nomme ces auteurs-là, c'est parce que ce sont tous des auteurs auxquels je reviens constamment, mes livres de chevet. Lorsque je suis déprimé, mon réflexe c'est de sortir un vieux Sartre, un Beckett. II y a des auteurs auxquels je reviens constamment et qui sont des auteurs très peu lus maintenant ; il y a une certaine veine de Paul Morand que j'aime assez, celle de Montociel par exemple; et puis Marcel Jouhandeau, Montherlant... II doit y avoir peu de gens sur terre qui ont lu, comme moi, tout Montherlant. C'est, avec Beckett, un des rares auteurs dont j'ai à peu près les œuvres complètes, ça doit bien faire une cinquantaine de volumes sur les rayons de ma bibliothèque. Ce qui ne l'empêche pas de me porter sur les nerfs à un degré pas possible parfois. Mais si on se met à vouloir traduire la complexité de mes rapports à Montherlant, on n'est pas sorti de l'auberge. Et puis il y a Bernanos, que je trouve absolument admirable, celui de Monsieur Ouine à tout le moins. Mais c'est difficile de définir les auteurs qui nous ont 
le plus influencé, il y a toujours une part d'inaperçu là-dedans. Curieusement, c'est peut-être un poète qui a eu une des influences les plus profondes et décisives sur mon écriture : Rimbaud, le Rimbaud d'Une saison en enfer a été absolument déterminant pour moi. C'est un texte que j'ai dû lire une vingtaine de fois, depuis un quart de siècle.

- Vous m'avez cité beaucoup d'auteurs européens, mais qu'en est-il des auteurs québécois?

- Il y a eu Réjean Ducharme. C'est un authentique créateur sur le plan du langage, même si parfois à mon sens il a écrit des livres peut-être un peu plus faciles que d'autres. II y a eu Hubert Aquin aussi. Ce qui était fascinant pour le jeune homme que j'étais c'est que c'était un grand intellectuel, c'était quelqu'un qui avait une approche de haute civilisation, tout à fait inusitée au Québec. Aquin pouvait réfléchir sur les oeuvres de St-Thomas D'Aquin. II pouvait construire des fictions autour de figures de la haute Renaissance. II apportait, il ouvrait une possibilité vers la culture universelle qu'on n'avait pas connue auparavant au Québec. À ce titre-là, même si ses oeuvres ne sont pas toutes absolument réussies, je pense qu'Hubert Aquin a droit à toute notre reconnaissance. Mais en dehors de ces deux auteurs là, j'ai le regret de dire que j'ai peu ...

\section{- Ferron?}

- Ce sont des auteurs que j'estime. Ferron, je le relisais justement avanthier. Je relisais le texte qu'il a écrit sur Claude Gauvreau, qui est un beau texte. Un homme qui savait écrire, Ferron. Évidemment, ma grande amie, Marie-Claire Blais, qui est comme à part pour moi. Elle n'a pas eu véritablement une influence sur moi, mais j'ai toujours eu énormément d'estime pour ce qu'elle faisait et depuis qu'on est devenus amis... La personne est à la hauteur de l'écrivain chez Marie-Claire.

- Bataille parlait souvent de l'être humain comme étant un être blessé : tout être humain a une fêlure, une faille. Est-ce que chez Gaétan Soucy, le besoin d'écrire, la nécessité d'écrire vient d'une blessure?

- Très certainement. Mais c'est une blessure qui est un peu comme l'horizon : c'est à la fois constamment là, ça nous englobe tout le temps, mais on ne peut jamais mettre le doigt dessus, on ne peut pas mettre le doigt sur I'horizon... De quel ordre est cette blessure? Je l'ignore. II est certain que je suis quelqu'un de blessé, mais comme tout le monde... Comme tout le 
monde, mais en même temps, pour revenir à notre point de départ, pour que se produise un tel investissement - un aussi exclusif investissement dans l'ordre de l'écrire - , cela demande une fêlure quelque part qui remonte sans doute très profondément. J'ai un souvenir, qui est peut-être un souvenir-écran, mais il me semble que c'est le plus vieux souvenir que j'ai, le souvenir dont je peux maîtriser les contours. Je devais avoir quatre ans, trois ans et demi peut-être. À la télévision, quelqu'un avait dû parler du plus beau jour de sa vie. J'avais descendu l'escalier derrière chez moi, il faisait un grand soleil, il me semble que c'était un samedi après-midi. Je m'étais dit - c'est curieux dans la tête d'un enfant de trois ans et demi, quatre ans - : "c'est le plus beau jour de ma vie". L'impression de bonheur que j'avais eue, je ne peux encore en parler maintenant sans réprimer un mouvement qui me ferait pleurer. Le sentiment d'intensité de bonheur a été tel que j'ai l'impression d'avoir été foudroyé à ce moment-là. II y a quelque chose qui a carbonisé en moi. Bien que je ne sache pas indiquer exactement par quel méandre, il me semble qu'il y a tout de même un lien étroit et souterrain profond entre cet instant-là et mon investissement total dans l'écriture. C'est curieux, mais je le dis vraiment le plus sincèrement du monde. C'est comme ça que cela m'apparaît.

- Écrire, cela nous permet-il d'espérer lorsqu'on voit cette humanité, les inégalités, lorsqu'on voit que le bien-vivre ensemble est difficile?

- II y aurait deux choses à dire principalement. D'abord, rappeler la phrase de Camus qui disait : «une littérature désespérée est une contradiction dans les termes". Ensuite, la littérature, l'écriture n'est pas là pour résoudre les problèmes du monde, elle est là pour exprimer le malaise à être. Tous ceux qui donnent une finalité à l'écriture sont toujours obligés d'admettre cette chose absurde à savoir que la littérature c'est terminé. Cela a été le cas avec Hegel qui avait assigné une certaine finalité à l'art; il dit : comme le concept maintenant a rejoint ce que l'art a essayé de rejoindre, l'art est maintenant chose du passé. Ou même René Girard qui disait que la vocation du littéraire c'était justement de nous révéler ce caractère mimétique, mais maintenant que le concept - encore une fois - a rejoint ce que tentait de rejoindre l'écriture romanesque, celle-ci se voit dépassée. Ce que Hegel ou M. Girard - que j'admire beaucoup - semblent oublier c'est qu'on continue d'être vivant et on continue d'éprouver le malaise, le magnifique malaise d'être et que cela appelle à l'expression. La littérature n'a pas à résoudre des problèmes, n'a pas à promettre quelque chose, 
mais a à dire le fondamental malaise de l'être humain. Et s'il advenait qu'on empêche les hommes d'exprimer ce malaise d'être, on serait en enfer, on serait dans la pire des sociétés. De toute façon, le fait qu'on continue d'écrire, de lire ce qui s'écrit ne peut que contribuer à une relation meilleure de l'homme à l'homme et de l'homme au monde. Enfin, à tout le moins, ce qu'on peut dire c'est que si on enlève cela, véritablement il ne nous restera plus rien...

- Aucun espoir...?

- Aucun espoir.

- Bon. Et vos projets, pour en finir?

- Je n'ai pas de projets pour en finir, ne criez pas victoire trop vite! J'espère bien pouvoir encore écrire au moins pour les trente prochaines années. Je dois tout de même vous avouer qu'en ce moment je suis dans une période d'incertitude due à des conjonctures très précises qui font que pour les quelques semaines à venir j'aurai peu l'occasion de me concentrer sur moi-même, de me recueillir. Mais, j'ai bon espoir que quelque chose d'inattendu est devant moi et façonnera mon avenir. (Après un silence) N'empêche que j'adorerais qu'une fée me fasse lire aujourd'hui ce que je vais écrire dans vingt-cinq ans.

\section{7 juin 1999}

Saint-Bruno-de-Montarville 